\title{
LA RIFORMA DELLA SCUOLA ITALIANA QUALE MODELLO PER LA RIFORMA CURRICOLARE IN CROAZIA
}

\author{
Debora Radolović \\ Scuola media superiore italiana Dante Alighieri, Pola
}

\section{Riassunto}

Il Governo croato sta continuando a lavorare alla Riforma curricolare e a capo della Commissione per l'attuazione della stessa è stato eletto il premier Plenković per garantire la continuità.

Il Governo italiano ha approvato la Legge sulla Riforma del sistema nazionale di istruzione e formazione, la cosiddetta Buona Scuola. Sono stati chiaramente definiti gli scopi, gli obiettivi, gli strumenti, le modalità di attuazione e valutazione, i bonus per docenti e gli school bonus per coloro che vogliono investire nell'infrastruttura scolastica.

Sono due mondi scolastici molto simili che abbisognano di un forte sostegno della società.

Parole chiave: riforma scolastica, riforma curricolare, autonomia, creatività, crescita 


\section{Introduzione}

La realtà scolastica ha bisogno di continui cambiamenti, miglioramenti ed evoluzione perché rappresenta il migliore investimento per il futuro.

È notevole l'impegno di due stati quali la Croazia e l'Italia in quanto si ritrovano a dover cambiare il Mondo Scuola onde renderlo più competitivo ed interessante al mondo del lavoro. La scuola in territorio europeo è complessa; molti stati hanno avviato le riforme scolastiche da diversi anni, dimostrando che bisogna cambiare il percorso didattico, rendendolo più dinamico, interessante e autonomo.

\section{La scuola in Croazia}

Quando ci troviamo ad analizzare la programmazione didattica di ogni singolo piano operativo di materia (curricolo) per lo stato croato significa dover seguire ciecamente un programma ministeriale che a volte si ritrova a essere formattato negli anni '60! La stessa cosa succede per molti indirizzi di studio, p. es. il programma triennale di Automeccanico, attualmente in vigore, è stato formulato nel 1964.

Le istituzioni scolastiche della Croazia si sono persino dovute ingaggiare per cercare il suddetto programma dato che l'Agenzia preposta (Agencija za strukovno obrazovanje i obrazovanje odraslih) non aveva depositato nel proprio archivio il programma classico in vigore in molte scuole di indirizzo industriale - artigianale.

Uno dei punti forti della Riforma curricolare proposta è quello di sviluppare il pensiero critico: creare allievi creativi che imparano a pensare.

Gli scopi della Riforma curricolare sono volti ad avviare il metodo di lavoro partecipativo attraverso il costante dialogo tra docenti, studenti e genitori. Nell'autunno 2014 il Ministero dell'Educazione e dell'Istruzione aveva bandito una gara per selezionare la proposta migliore per riformare totalmente i curriculum scolastici in Croazia. Boris Jokić è risultato il vincitore sebbene fosse l'unico partecipante. Nei mesi seguenti si sono formati team di esperti di tutte le discipline scolastiche, col compito di definire le linee guida della riforma, nonché il metodo di lavoro. Aveva 
seguito a ciò il lavoro di un Comitato di dieci esperti per redigere la riforma. In quel momento il Comitato ha chiamato a Zagabria più di 900 istituzioni della verticale scolastica e 64.000 docenti, che hanno contribuito alla stesura dei diversi documenti attraverso vari incontri sui macro-punti. Dopo sedici mesi di lavoro erano pronti i 52 documenti del nuovo sistema scolastico e vennero presentati pubblicamente ai numerosi seminari di aggiornamento.

Attualmente, il Ministero croato sta cercando di ripristinare le commissioni preposte ai vari curricoli e ciò significa che nei prossimi mesi ci saranno sicuramente nuove proposte mentre le modifiche di legge dovranno aspettare tempi migliori. Nel frattempo, per appacificare le varie correnti politiche, è stato scelto il premier Plenković in veste di presidente della Commissione per l'attuazione della Riforma curricolare.

\section{Gli scopi della riforma scolastica in Italia}

Il 13 luglio 2015, sulla Gazzetta Ufficiale è stata pubblicata la Legge n. 107 cioè la Riforma del sistema nazionale di istruzione e formazione (con delega per il riordino delle disposizioni legislative vigenti), conosciuta come La Buona Scuola dell'allora premier Renzi.

Cercando di riassumere in breve gli scopi della Riforma si può dire che offre alle scuole di attuare totalmente l'autonomia già presente nella realtà italiana, permette di innalzare i livelli d'istruzione e le competenze, contrastando le disuguaglianze, prevenendo e recuperando l'abbandono scolastico, realizzando una scuola aperta che garantisca il diritto allo studio e pari opportunità di successo formativo.

Nello specifico alle Istituzioni scolastiche viene garantita la partecipazione alle decisioni degli Organi Collegiali, l'organizzazione è orientata alla flessibilità, alla diversificazione, all'efficacia e all'efficienza del servizio scolastico. Inoltre, viene consentito un miglior utilizzo delle risorse e delle strutture, con un particolare accento all'introduzione di nuove tecnologie e al coordinamento con il contesto territoriale. Per quanto riguarda l'autonomia scolastica, essa presuppone una scuola come servizio pubblico per la persona e la comunità e ciò comporta una riorganizzazione del sistema e un cambiamento di approccio alla formazione come processo di apprendimento. Infatti, l'autonomia scolastica deve essere funzionale alle 
esigenze didattiche, organizzative e progettuali onde ottenere il successo formativo.

Gli strumenti che vengono proposti hanno un ruolo sinergico: è fondamentale il ruolo del Dirigente scolastico e dello staff in quanto attuano attività di coordinamento che trovano nel Piano dell'Offerta Formativa (POF) triennale, il documento fondamentale dell'identità della scuola che esplica l'intera progettazione, nella quale il Dirigente Scolastico fa le scelte d'indirizzo strategico, quali le attività, le scelte di gestione. Il POF viene elaborato rispettivamente deliberato dal Collegio docenti ed approvato dal Consiglio d'Istituto e può venir ritoccato annualmente.

Gli altri strumenti della Riforma richiedono la partecipazione di tutti gli elementi come gli organi collegiali, gli studenti e le famiglie. Bisogna pure interagire con il territorio e un ruolo fondamentale in questo senso lo svolge il Direttore dei servizi generali e amministrativi (DSGA) che sovrintende ai servizi generali amministrativo-contabili e ne cura lorganizzazione svolgendo funzioni di coordinamento, promozione delle attività e verifica dei risultati. Inoltre, organizza autonomamente l'attività del personale amministrativo, tecnico e ausiliario (ATA) nell'ambito delle direttive del Dirigente scolastico. Quest'ultimo tassello ha un compito fondamentale nell'ambito dell'organico dell'autonomia che le istituzioni scolastiche adottano ogni anno, con modalità organizzativa coerente con gli obiettivi generali e il miglioramento dell'offerta formativa.

La scuola autonoma ha una particolare necessità di procedere con una formazione permanente del personale volta in particolar modo all'innovazione tecnologica ma non solo. C'e' bisogno pure di strumenti di valutazione, rendicontazione, trasparenza e pubblicità, che devono venir realizzati grazie al coordinamento di tutto il personale scolastico. Per quanto riguarda il percorso formativo degli studenti, la novità che ha destato più interesse è quella relativa agli studenti delle superiori: è stata decretata l'alternanza scuola - lavoro, una rotazione che incrementa le opportunità di lavoro e li orienta nelle scelte future. L'alternanza ha stabilito complessivamente 200 ore obbligatorie nei Licei e almeno 400 ore obbligatorie negli Istituti Tecnici e Istituti Professionali. Le ore di lavoro si realizzano nel corso del secondo biennio e nell'ultimo anno della scuola secondaria di secondo grado a partire dall'anno scolastico 2015/2016, 
indicando la durata complessiva di almeno 400 ore per gli istituiti tecnici e professionali e di almeno 200 ore per i licei; autorizza la spesa di 100 milioni di euro annui a decorrere dall'anno 2016; istituisce il Registro nazionale per l'alternanza scuola-lavoro, a cui si devono iscrivere le imprese e gli enti pubblici e privati disponibili ad accogliere studenti.

L'alternanza scuola lavoro diventa così una strategia educativa ove l'impresa e l'ente pubblico o privato sono invitati ad assumere un ruolo complementare all'aula e al laboratorio scolastico nel percorso di istruzione degli studenti in modo da contribuire alla realizzazione di un collegamento organico tra istituzioni scolastiche e formative e il mondo del lavoro ${ }^{[1]}$.

Oltre all'alternanza scuola lavoro, la Legge si occupa di sviluppare e migliorare le competenze digitali e rendere la tecnologia uno strumento didattico. Per questo motivo è stato realizzato un Piano Nazionale per la scuola digitale che offre agli studenti attività volte allo sviluppo di competenze digitali, formando docenti e personale ATA preparati all'innovazione didattica e abili a sviluppare la cultura digitale. Nell'ambito dell'organizzazione del Piano Nazionale si offrono strumenti organizzativi e tecnologici per favorire il controllo, la trasparenza, la condivisione dei dati e lo scambio di informazioni. Sono previsti investimenti statali per gli strumenti didattici e i laboratori per migliorare la formazione e l'innovazione oltre che per l'infrastruttura dove si prevede il potenziamento delle infrastrutture di rete.

Un argomento a se stante è quello del Dirigente scolastico (DS) che garantisce un'efficace ed efficiente gestione delle risorse umane, finanziarie, tecnologiche e materiali assicurando il buon andamento dell'Istituzione Scolastica. Il DS svolge i compiti di direzione, gestione, organizzazione e coordinamento e ha la responsabilità della gestione delle risorse finanziarie e strumentali e dei risultati del servizio come pure della valorizzazione delle risorse umane. Gli obblighi del DS sono definiti nel Decreto legislativo n. 165/01 e si possono riassumere brevemente con le seguenti parole d'ordine: promuovere, organizzare, valorizzare, garantire la legittimità, gestire. Il lavoro del DS viene valutato ${ }^{[2]}$ ed in primo luogo vengono valutate le competenze gestionali ed organizzative, la correttezza, la trasparenza,

[1] https://scuolalavoro.registroimprese.it/rasl/legge?2 (8/1/2018)

[2] http://www.istruzione.it/snv/allegati/2016/ds36_16.pdf (8/1/2018) 
l'efficacia e l'efficienza e questi parametri danno il proprio contributo ai risultati di miglioramento previsti dal Rapporto di Autovalutazione (RAV).

Per quanto riguarda i docenti, essi devono aggiornarsi continuamente e la formazione in servizio è obbligatoria, permanente e strutturale. A livello statale esiste il Piano Nazionale di Formazione che individua ogni tre anni le priorità nazionali per la formazione. L'attività di formazione delle scuole e l'utilizzo della Carta Docenti ${ }^{[3]}$ devono essere coerenti con il POF, con il Piano Nazionale di Formazione e con i risultati del Piano di Miglioramento (PDM).

Anche il lavoro dei docenti viene valutato, esiste un fondo ministeriale per la valorizzazione dei docenti. Il Comitato di valutazione è composto dal DS, da due docenti del Collegio Docenti, da un docente del Consiglio d'Istituto, da due genitori o da un genitore e uno studente e da un esterno (ispettore, dirigente o docente). Il Comitato individua i criteri per la valutazione dei docenti sulla base della qualità dell'insegnamento e del contributo al miglioramento della scuola, inoltre analizza i risultati inerenti all'innovazione didattica e metodologica e alla responsabilità nel coordinamento organizzativo, didattico e della formazione personale. Esprime inoltre il proprio parere sul superamento del periodo di formazione e prova per il personale docente ed educativo come pure, valuta il servizio del personale docente, su richiesta dell'interessato, previa relazione del DS ed esercita le competenze per la riabilitazione del personale docente.

Il progetto ministeriale del Portale Unico dei Dati della Scuola (Open Data) ${ }^{[4]}$ garantisce l'accesso e la riutilizzazione dei dati pubblici. Il Miur mette a disposizione dei cittadini in maniera trasparente l'accesso libero alle informazioni e ai dati della scuola senza autenticazione o identificazione, in conformità con la normativa vigente. È possibile vedere i bilanci delle scuole, i dati pubblici sul sistema nazionale di valutazione, l'anagrafe dell'edilizia scolastica, i provvedimenti di incarico di docenza, il POF, i dati dell'osservatorio tecnologico, i materiali didattici e le opere autoprodotti dagli istituti scolastici e rilasciati in campo aperto. Ci saranno anche i dati, i documenti e le informazioni per l'avanzamento didattico, tecnologico e d'innovazione del sistema scolastico, come pure l'identità

[3] https://cartadeldocente.istruzione.it/\#/ (è un'iniziativa del MIUR che istituisce la Carta elettronica per l'aggiornamento e la formazione dei docenti di ruolo delle istituzioni scolastiche, pari a 500 euro annui) (8/1/2018)

[4] http://dati.istruzione.it/opendata/ (8/1/2018) 
digitale di studenti e docenti.

Per quanto riguarda l'infrastruttura scolastica è previsto il cosiddetto school bonus cioè un'agevolazione fiscale per le donazioni ossia erogazioni liberali effettuate nei confronti degli istituti del sistema nazionale per la realizzazione di nuove strutture scolastiche, la manutenzione e il potenziamento di quelle esistenti ed il sostegno a interventi per il miglioramento dell'occupazione degli studenti.

\section{Conclusione}

La riforma scolastica in Croazia si sta riavviando, dopo un periodo di stasi dovuto alle agitazioni politiche presenti negli ultimi due anni. In questi giorni i colleghi delle istituzioni croate che facevano parte delle prime Commissioni impegnate nella stesura dei nuovi curricoli sono stati contattati e molti hanno accettato la collaborazione per la conclusione della proposta della Riforma curricolare. Per quanto riguarda l'Italia, si può dire che la Riforma scolastica è ben avviata sebbene sono presenti le dimostrazioni in piazza e altre forme di protesta civile. Per tutte e due le realtà ci si auspica una crescita e un miglioramento del Mondo Scuola, in quanto i risultati del test PISA-Invalsi 2015 vede l'Italia piazzata al 34esimo posto, sebbene i risultati dimostrano una forte differenza regionale. Per quanto riguarda la Croazia, essa si trova al 37esimo posto sui settanta paesi coinvolti. Sono questi gli argomenti più rilevanti che richiedono l'avvio della Riforma curricolare in Croazia al più presto e la rifinitura della Riforma scolastica in Italia.

\section{Bibliografia}

BATUR, M. (a cura di.), 2017, Novi horizonti strukovnoga obrazovanja: izvještaj vanjskoga vrednovanja eksperimentalne provedbe novih strukovnih kurikuluma, Nacionalni centar za vanjsko vrednovanje obrazovanja, Zagabria.

CALVINO, R. S., 2015, Organizzazione e legislazione scolastica dopo la Buona Scuola, Maggioli Editore, Santarcangelo di Romagna. 
Siti web

https://cartadeldocente.istruzione.it/\#/ (è un'iniziativa del MIUR che istituisce la Carta elettronica per l'aggiornamento e la formazione dei docenti di ruolo delle istituzioni scolastiche, pari a 500 euro annui) $(8 / 1 / 2018)$

http://dati.istruzione.it/opendata/ (8/1/2018)

http://www.istruzione.it/snv/allegati/2016/ds36_16.pdf (8/1/2018)

http://www.kurikulum.hr/okvir-nacionalnog-kurikuluma-onk/ $(8 / 1 / 2018)$

https://scuolalavoro.registroimprese.it/rasl/legge?2 (8/1/2018) 


\section{Reforma talijanske škole kao model za kurikularnu reformu u Hrvatskoj}

Hrvatska Vlada nastavlja kontinuirani rad s ciljem kurikularne reforme na čelu s premijerom Plenkovićem koji je postavljen da bi se osigurao nastavak rada.

Talijanska Vlada je usvojila Zakon o reformi nacionalnoga odgoja i obrazovanja, tzv. „Dobra škola“. Jasno su bili definirani svrha i ciljevi, alati, načini realizacije i vrednovanja te dodaci za rad za nastavnike i školu te za sve one koji žele ulagati u školsku infrastrukturu. Postoje dvije vrlo slične realnosti u školama koje imaju potrebu za snažnom podrškom društva.

Ključne riječi: školska reforma, kurikularna reforma, autonomija, kreativnost, razvoj

\section{The Reform of Italian Schools as a Model for Curriculum Reform in Croatia}

The Croatian government has continued with its work aiming at curriculum reform with premier Plenković at its head: he has been given this task in order to ensure that this reform will continue.

The Italian government has incorporated a law concerning the reform of national education tiltled as the „Good School“. The aims and means have been clearly defined, the tools required, the means of realisation and evaluation, as well as additions for teachers, schools and all those who desire investing in school infrastructure. There are two very similar realities in schools that have the need for the strong support of the community.

Key words: school reform, curriculum reform, autonomy, creativity, development 